\title{
Vanillin Determination in Food Products with Pd Nanoparticles Modified Poly(Methylene Blue) Film Electrode
}

\author{
Çağrı Ceylan Koçak ${ }^{*}$ \\ Bergama Vocational School, Dokuz Eylül University, İzmir, Turkey \\ *ceylan.kocak@deu.edu.tr \\ Received: 17 February 2019 \\ Accepted: 08 May 2019 \\ DOI: $10.18466 /$ cbayarfbe. 528144
}

\begin{abstract}
Here, metal nanoparticles modified conductive polymer film electrode was fabricated via electrochemical technique. Methylene blue was electrochemically polymerized on the bare glassy carbon electrode surface. Then palladium nanoparticles were modified on the polymer surface by consecutive potential cycles. Resulting composite electrode was characterized with scanning electron microscopy and electrochemical impedance spectroscopy. Palladium nanoparticles modified poly(methylene blue) film glassy carbon electrode was used for sensitive and selective determination of vanillin with two linear ranges between $0.02-1 \mu \mathrm{M}$ and $2-50 \mu \mathrm{M}$ and a limit of detection as $0.01 \mu \mathrm{M}$. Proposed electrode accurately determine vanillin content in commercial biscuit and cake samples.
\end{abstract}

Keywords: Vanillin, Pd Nanoparticles, Methylene Blue, Modified Electrode, Voltammetry.

\section{Introduction}

4-hydroxy-3-methoxybenzaldehyde, known as vanillin $\left(\mathrm{C}_{8} \mathrm{H}_{8} \mathrm{O}_{3}\right)$ is a phenolic compound that is found naturally in vanilla seeds [1]. Vanillin (VAN) is the most commonly ingredient that responsible from flavoring in beverages and foods, used in perfumery and cosmetic industry, and also in pharmaceutical aids [2]. VAN extract from natural seeds is expensive that synthetic form was used in many industries. The excess use of VAN led to various diseases such as headache and kidney problems [3]. Therefore, it is significant to determine its amount both for health issues and control the quality of foods.

VAN can be determined with capillary electrophoresis [4], liquid chromatography-quadrupole linear ion trap mass spectrometry [5] and chemiluminometry [6]. In spite of the fact that these techniques require time consuming sample preparation step and operational expertise. Besides they constructed expensive equipment and consume lots of reagents. Electrochemical techniques overcome these mentioned disadvantages of other technique with its simplicity, fast response, and low costed equipment. Sensitivity of the electrochemical techniques are mainly affected by the electrode type that many researchers used modified electrodes to enhance the poor response of conventional electrodes towards analytes [7]. For this purpose; conductive polymers [8] and metal nanoparticles [9] were widely used.

Methylene blue (MB), (3,7bis(dimethylamino)phenothiazine), is a polycyclic structure that contains sulphur and nitrogen atoms. It is the member of phenothiazine family and known as a common organic dye which is water soluble [10]. MB is also used for electrode modification due to its polymerization ability, strong adherence to the surface that led a stable modifier and high conductivity that promote electron transfer [11]. Polymerized MB film electrodes were used for determination of wide range of organic/inorganic molecules such as dopamine [12], ascorbic acid [13], sulphide [14].

Metal nanoparticles attracted significant interests for various fields of research area due to their large surface area, ability to promote electron transfer rate and good catalytic activity that reduce over-potential [15]. Pd is one of the most studied metal particles, among all kind of nanoparticles. Pd nanoparticles were used in determination of food additives, antidepressants, detection of hydrogen peroxide, and catalysis of methanol and ethanol oxidation in fuel cells, promotion of methane oxidation $[16,17]$.

Here, palladium nanoparticles decorated conductive methylene blue polymer was prepared by two-step for highly sensitive determination of VAN. In first step, MB monomer was electrochemically polymerized on the glassy carbon electrode surface and then $\mathrm{Pd}$ nanoparticles were modified on the polymer film via electrochemical deposition technique by cyclic voltammetry in the second step. Resulting composite surface enhanced the electrocatalytic activity of bare electrode towards VAN that has high stability and sensitivity. Moreover, proposed electrode provides accurate determination of VAN in different food products. 


\section{Materials and Methods}

\subsection{Reagents and Apparatus}

$\mathrm{PdCl}_{2}, \mathrm{H}_{2} \mathrm{SO}_{4}$ and $\mathrm{HCl}$ were supplied from Merck. All reagents used without further purification. Milli-pore ultra-pure water was used for dilution of the solutions. $\mathrm{pH}$ of PBS solution was adjusted with $\mathrm{NaOH}$.

An Autolab electrochemical system (Metrohm) equipped with a NOVA software was used for voltammetric studies. All measurements were carried out in a three-electrode system consisting of; a platinum wire as a counter electrode, $\mathrm{Ag} / \mathrm{AgCl} / \mathrm{KCl}$ as a reference electrode, and glassy carbon electrode (GCE) as a working electrode. The electrodes were characterized with scanning electron microscope (SEM; JEOL JSM7500F)

\subsection{Preparation of Commercial Food Samples}

Commercial biscuit and cakes were obtained from a local market. Each food samples were ground in a mortar until homogenized particles were obtained. $0.5 \mathrm{~g}$ of this resulting powder of each biscuit and cake was mixed with $10.0 \mathrm{~mL}$ ethanol. The mixture was mechanically shaken for $90 \mathrm{~min}$ and then centrifugated for $20 \mathrm{~min}$ at $4000 \mathrm{rpm}$. Finally, above of this solution tube was used for VAN determination.

\section{Results and Discussion}

\subsection{Preparation and Characterization of Electrode}

Fig.1a shows the polymerization of MB monomer on bare GCE surface in alkaline media. Polymerization potential window was selected as wide as possible to see the all oxidation and reduction peaks. Potential cycles were applied between -0.7 to $+1.2 \mathrm{~V}$ in PBS (pH 10) containing $2 \mathrm{mM} \mathrm{MB}$ monomer. Cationic radical species were formed at higher potentials $(1.1 \mathrm{~V})$ which were necessary for polymerization. In addition, an anodic peak $(-0.13 \mathrm{~V})$ and cathodic peak $(-0.35 \mathrm{~V})$ were observed in the first cycle. By the formation of polymeric film on the electrode surface, the anodic peak was decrease while cathodic peak was slightly shifted to negative values. With the consecutive cycles, another redox pair was observed. These new pair of peaks reduced at $-0.10 \mathrm{~V}$, while oxidized at more positive potentials with successive cycles. The successful polymerization was occurred at GCE surface by electropolymerization technique which is also confirmed with scanning electron microscopy and electrochemical impedance spectroscopy techniques. In addition, $\mathrm{Pd}$ nanoparticles were deposited on the PMB/GCE surface by cyclic voltammetry that was presented in Fig.1b. PMB/GCE was immersed in the 1 $\mathrm{mM} \mathrm{Pd}{ }^{2+}$ containing $\mathrm{H}_{2} \mathrm{SO}_{4}$ solution and ten repetitive cycles were applied between $+0.30 \mathrm{~V}$ and $-0.75 \mathrm{~V}$. Both anodic and cathodic peaks were increased with the increase in the potential cycles that depicts the formation of $\mathrm{Pd}$ nanoparticles on the polymer film surface.
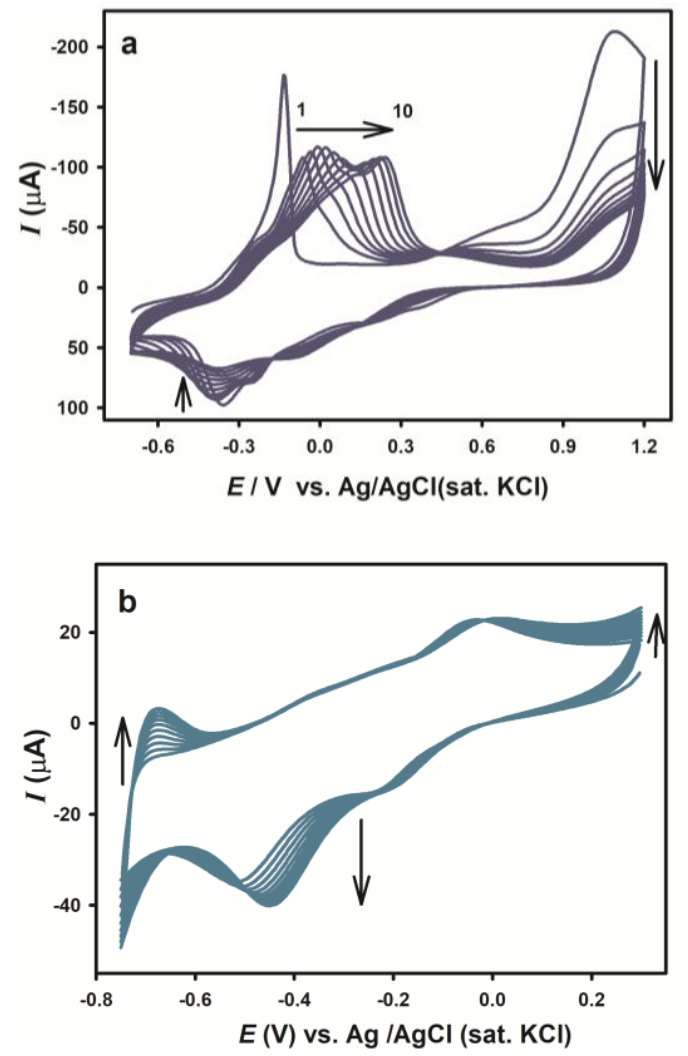

Figure 1. a) Polymerization of $2 \mathrm{mM} \mathrm{MB}$ on GCE in $\mathrm{pH} 10 \mathrm{PBS}$ with $0.1 \mathrm{~V} \mathrm{~s}^{-1}$ scan rate. b) $\mathrm{CVs}$ of palladium nanoparticles deposition on $\mathrm{PMB} / \mathrm{GCE}$.

The morphologies of bare GCE, PMB/GCE and $\mathrm{Pd} / \mathrm{PMB} / \mathrm{GCE}$ were recorded by scanning electron microscopy (SEM). A smooth and uniform surface was observed for bare GCE (Fig. 2a). It can be directly observed that, bud like shapes formed on the bare surface after the electropolymerization MB (Fig.2b). The buds on the surface was growth with the Pd nanoparticles deposition and EDX analysis confirms the existence of $\mathrm{Pd}$ content (\%13 weight gain) on the Pd/PMB/GCE surface (Fig.2c). Physical and interfacial properties of the bare and modified electrodes were characterized with electrochemical impedance spectroscopy (EIS). Spectra was performed in $5 \mathrm{mM}$ $\mathrm{K}_{3}\left[\mathrm{Fe}(\mathrm{CN})_{6}\right] / \mathrm{K}_{4}\left[\mathrm{Fe}(\mathrm{CN})_{6}\right]$ containing $0.1 \quad \mathrm{M} \quad \mathrm{KCl}$ solution. Frequencies between 5 to $75000 \mathrm{~Hz}$ were applied at GCE, PMB/GCE, Pd/PMB/GCE and comparative results were represented in Fig. $2 \mathrm{~d}$. The impedance circuit contains the solution resistance (Rs), , constant phase element ( $\mathrm{CPE}$ or $\mathrm{Q})$, and charge transfer resistance (Rct). In Nyquist diagrams, a straight line with a slope of nearly $45^{\circ}$ is related to the mass transport process and semicircle diameter at high frequencies relates to the resistance characteristics of the electrode/electrolyte interface. A large semicircle related to the highest the charge transfer resistance was obtained for bare GCE (380 $\Omega$, curve $i)$. An obvious decrease in resistance observed for curve ii (95 $\Omega$ ) indicates the formation of conductive PMB on the bare GCE surface that could accelerate the electron transfer 
rate. After the $\mathrm{Pd}$ nanoparticles deposition, the resistance of the electron transfer was significantly reduced (25 $\Omega$, curve iii). Overall results depict the formation of both Pd nanoparticles and PMB film on the bare GCE surface.

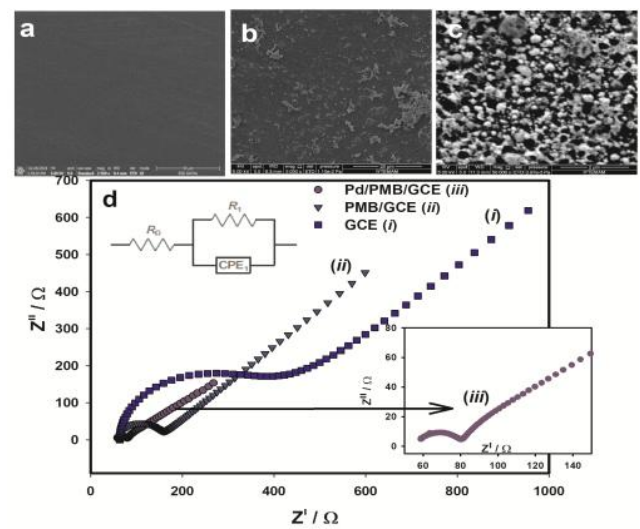

Figure 2. SEM images of a) Bare GCE, b) PMB/GCE, c) $\mathrm{Pd} / \mathrm{PMB} / \mathrm{GCE}$ and d) EIS spectra of electrodes. Inset contains related circuit and zoomed plot of $\mathrm{Pd} / \mathrm{PMB} / \mathrm{GCE}$.

\subsection{Voltammetric Behavior of VAN}

The electrochemical behaviour of VAN was investigated at different electrodes using cyclic voltammetry to understand the effect of modifiers. Fig. 3 depicts the $\mathrm{CVs}$ of GCE, PMB/GCE, and $\mathrm{Pd} / \mathrm{PMB} / \mathrm{GCE}$ at $0.1 \mathrm{mM} \mathrm{VAN}$ containing $\mathrm{pH} 6$ buffer solution. The quasi-reversible redox behavior observed for VAN at all electrodes. Bare GCE has very poor signal $(3 \mu \mathrm{A})$ indicating slow electrode kinetics. After the polymerization of MB on GCE surface, peak current significantly enhanced $(12.3 \mu \mathrm{A})$. VAN oxidation was observed at $0.80 \mathrm{~V}$ at both $\mathrm{PMB} / \mathrm{GCE}$ and $\mathrm{Pd} / \mathrm{PMB} / \mathrm{GCE}$. Another small oxidation peak was observed at $0.83 \mathrm{~V}$ in the reverse scan that can be attributed to the re-oxidation of adsorbed VAN compounds on the electrode surface. Morover, the highest peak current was obtained at $\mathrm{Pd} / \mathrm{PMB} / \mathrm{GCE}$ $(39.3 \mu \mathrm{A})$ that can be attributed to the synergistic effect of metal nanoparticles and conductive polymer. The improved peak characteristics in terms of peak current was obtained for proposed $\mathrm{Pd} / \mathrm{PMB} / \mathrm{GCE}$ which is 13 times higher than bare GCE and 3 times higher than PMB/GCE.

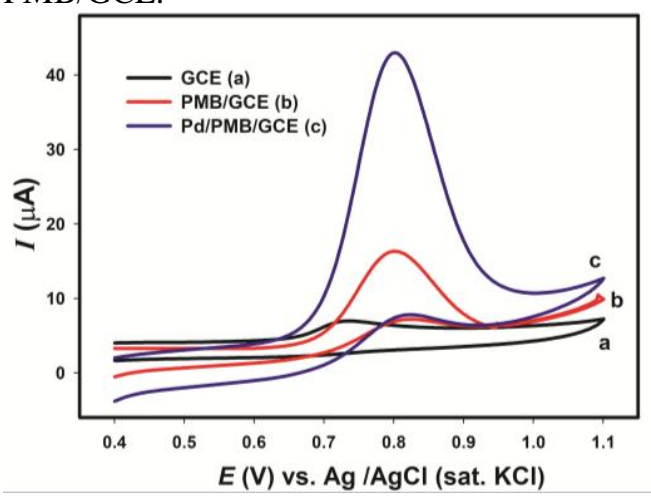

Figure 3. $\mathrm{CVs}$ of $0.1 \mathrm{mM}$ VAN in $\mathrm{pH} 6 \mathrm{PBS}$ with 0.05 $\mathrm{V}$ s-1 scan rate at a) GCE. b) PMB/GCE and c) $\mathrm{Pd} / \mathrm{PMB} / \mathrm{GCE}$

The effect of $\mathrm{pH}$ on the VAN behavior was studied by $\mathrm{CV}$ in $0.1 \mathrm{M}$ buffer solution with various $\mathrm{pH}$ values (from 2 to 8) (not shown here). A linear relationship between the peak potential and the $\mathrm{pH}$ was observed at $\mathrm{Pd} / \mathrm{PMB} / \mathrm{GCE}$. The slope of related plot is close to the theoretical value of $59 \mathrm{mV} / \mathrm{pH}$ that suggested as electrochemical reaction of VAN occurred with twoproton/ two-electron transfer. In addition, the highest peak current was obtained at $\mathrm{pH} 6$ was chosen as an optimum value for further studies. Moreover, to obtain more sensitive determination results, cycle number of polymerization, monomer and palladium concentration and Pd deposition cycle number were also optimized. Considering the overall results, parameters were optimized as follows; monomer concentration: $2 \mathrm{mM}$, cycle number of polymerization; 20, Pd concentration; 1 $\mathrm{mM}$ and cycle number of $\mathrm{Pd}$ deposition; 10. These optimized parameters were used for further studies.

In order to understand the surface reaction was controlled by diffusion or adsorption, VAN oxidation was investigated with various scan rates $(10-250 \mathrm{mV} \mathrm{s}$ $\left.{ }^{1}\right)$. Based on these data, Ipa versus square root scan rate $\left(v^{1 / 2}\right)$ graphs were plotted for bare GCE, PMB/GCE and $\mathrm{Pd} / \mathrm{PMB} / \mathrm{GCE}$ and it was presented in Fig. 4. VAN peak current was increased at all electrodes with increasing the scan rate. The slope value was getting higher with the electrode modification as expected. According to the results, it can be said that electrode reaction was all controlled by diffusion at each electrode.

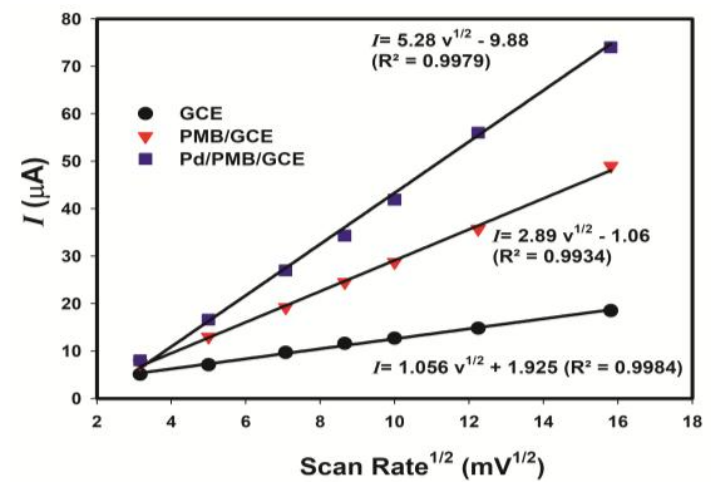

Figure 4. Dependence of VAN peak current on square root of scan rate at different electrodes.

Differential pulse voltammetry (DPV) was selected for determination of VAN by using $\mathrm{Pd} / \mathrm{PMB} / \mathrm{GCE}$ under the optimized experimental conditions (pulse amplitude: $50 \mathrm{mV}$, pulse time: $30 \mathrm{~ms}$, scan rate: $10 \mathrm{mV} \mathrm{s}^{-1}$ ). Fig. 5 exhibits the DP voltammograms of increasing concentrations of VAN at prepared electrode. Peak current of VAN was proportional with its concentration in the ranges of $20 \mathrm{nM}-1 \mu \mathrm{M}$ and $2 \mu \mathrm{M}-50 \mu \mathrm{M}$ with two segments. The related calibration graph was located in the inset of Fig.6. The linear regression equations were found as Ipa $(\mu \mathrm{A})=10.43 \mathrm{C}_{\mathrm{VAN}}(\mu \mathrm{M})+1.83$, $\left(\mathrm{R}^{2}\right.$ 
$=0.9941)$, and Ipa $(\mu \mathrm{A})=0.260 \mathrm{C}_{\mathrm{VAN}}(\mu \mathrm{M})+12.35,\left(\mathrm{R}^{2}\right.$ $=0.9957)$. Calculated detection limit was $10 \mathrm{nM}(\mathrm{S} / \mathrm{N}=$ 3). The proposed $\mathrm{Pd} / \mathrm{PMB} / \mathrm{GCE}$ has relatively lower detection limit value than those previously published $\begin{array}{llll}\text { literatures as in Table } 1 & \text { [27-29]. }\end{array}$

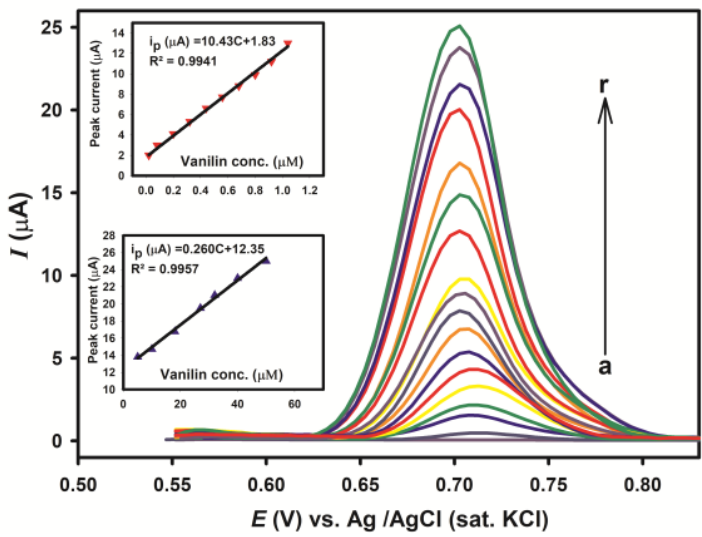

Figure 5. $\mathrm{DPV}$ s of $\mathrm{Pd} / \mathrm{PMB} / \mathrm{GCE}$ with increasing concentrations. (a: blank solution, b to $\mathrm{r}$ : $20 \mathrm{nM}$ to 50 $\mu \mathrm{M}$ VAN). Insets are related calibration graphs.

Interference study gave information about selectivity of the proposed method. Herein, various interfering

Table 1. Comparison of literature results with the proposed method. substances (benzoic acid, glucose, , sucrose, oxalic acid, caffeic acid, tartaric acid, citric acid) and some ions such as $\mathrm{Zn}^{2+}, \mathrm{Al}^{3+}, \mathrm{Mg}^{2+}, \mathrm{Na}^{+} \mathrm{Fe}^{3+} ; \mathrm{Pb}^{2+}, \mathrm{K}^{+}, \mathrm{Cl}^{-}, \mathrm{NO}_{3}{ }^{-}$, $\mathrm{SO}_{4}^{2-}$ was studied. 1000 fold excess of each substance has no significant effect on $5 \mu \mathrm{M}$ VAN $(\mathrm{RSD} \leq \% 5)$.

One of the main aims of using composite electrode is to enhance the oxidation current and stability of the electrode surface towards the VAN. Thus, oxidation peak current of VAN has been recorded daily for two week with the same electrode. Pd/PMB/GCE kept at above the supporting electrolyte solution in a close system that prevents the entrance of oxygen. At the end of two weeks, the electrode maintain $\% 97$ of its original signal towards VAN. It can be said that highly stable electrode surface was achieved with this study. Moreover, 10 different fabricated Pd/PMB/GCE were employed for VAN detection and RSD was calculated as $3.1 \%$. Repeatability studies of Pd/PMBGCE were utilized with measuring the VAN response of 50 repetitive measurements. Satisfactory results were obtained as $3.4 \%$ of RSD value.

\begin{tabular}{llll}
\hline Method & Linear range & LOD & Ref. \\
\hline Au-Pd/graphene/GCE & $0.1-7.0$ and $10.0-40.0 \mu \mathrm{M}$ & $0.020 \mu \mathrm{M}$ & {$[18]$} \\
Au/MWCNT/GCE & $0.02-6.5$ and $7.0-75 \mu \mathrm{M}$ & $0.038 \mu \mathrm{M}$ & {$[19]$} \\
Ag nanoplates graphene/GCE; & $2-100 \mu \mathrm{M}$ & $0.33 \mu \mathrm{M}$ & {$[20]$} \\
Pd/PMB/GCE & $\mathbf{0 . 0 2 - 1}$ and 2-50 $\boldsymbol{\mu M}$ & $\mathbf{0 . 0 1} \boldsymbol{\mu M}$ & This work \\
\hline
\end{tabular}

\subsection{Real Sample Assay}

The practical applicability of the proposed method was investigated with various food products at $\mathrm{Pd} / \mathrm{PMB} / \mathrm{GCE}$ via standard addition method. Commercial biscuit and cake samples pretreated according to the sample preparation method in Section 2.2 .

Table 2. Vanilin determination in different foods $(n=3)$.

\begin{tabular}{lcccc}
\hline Samples & $\begin{array}{c}\text { Added } \\
\boldsymbol{\mu M}\end{array}$ & $\begin{array}{c}\text { Found } \\
\boldsymbol{\mu M}\end{array}$ & $\begin{array}{c}\text { Recovery } \\
\mathbf{\%}\end{array}$ & $\begin{array}{c}\text { RSD } \\
\boldsymbol{\%}\end{array}$ \\
\hline Cake & 0 & 2.3 & - & 3.2 \\
& 4 & 6.1 & 96.8 & 2.7 \\
& 8 & 10.4 & 100.9 & 3.1 \\
Bisquite & 12 & 14.5 & 101.4 & 2.4 \\
& 0 & 2.5 & - & 2.3 \\
& 4 & 6.7 & 103.1 & 2.9 \\
& 8 & 10.3 & 98 & 3.2 \\
& 12 & 14.7 & 101.4 & 2.7 \\
\hline
\end{tabular}

The obtained results are given in Table 2 and recovery values are found in the range of $96.8 \%$ and $103.1 \%$. Vanillin content in biscuit and cake samples were found as $76.45 \mu \mathrm{g} / \mathrm{g}$ and $76.07 \mu \mathrm{g} / \mathrm{g}$, respectively. These high recovery values reveal that the developed composite electrode has accurately determine VAN content in commercial cake and biscuit samples.

\section{Conclusion}

In this study, electrodeposition of Pd nanoparticles on electropolymerized methylene blue film was successfully fabricated on glassy carbon electrode. Based on the results, modification of Pd nanoparticles on PMB film significantly increases the activity of electrode towards vanillin. The common interferants have no influence in the oxidation signal of VAN. The proposed electrode has two linear segments (20 nM- 1 $\mu \mathrm{M}$ and $2 \mu \mathrm{M}-50 \mu \mathrm{M}$ ) and low detection limit of 10 $\mathrm{nM}$ by DPV. In conclusion, the proposed method is a promising alternative for accurate and sensitive determination of VAN in various food products. 


\section{Ethics}

There are no ethical issues after the publication of this manuscript.

\section{References}

1. Ali HS, Abdullah AA, Pınar PT, Yardım Y, Şentürk Z. 2017. Simultaneous voltammetric determination of vanillin and caffeine in food products using an anodically pretreated boron-doped diamond electrode: Its comparison with HPLC-DAD. Talanta; 170: $384-391$

2. Almeida ARRP, Freitas VLS, Campos JIS, da Silva MDMCR, Monte MJS. 2019. Volatility and thermodynamic stability of vanillin. Journal of Chemical Thermodynamics; 128: 45-54.

3. Altunay N. Development of vortex-assisted ionic liquiddispersive microextraction methodology for vanillin monitoring in food products using ultravioletvisible spectrophotometry. 2018. LWT - Food Science and Technology; 93: 9-15.

4. Minematsu S, Xuan G-S, Wu X-Z. 2013. Determination of vanillin in vanilla perfumes and air by capillary electrophoresis. Journal of Environmental Sciences; 25: S8-S14.

5. Shen Y, Han C, Liu B, Lin Z, Zhou X, Wang C, Zhu Z. 2014 Determination of vanillin, ethyl vanillin, and coumarin in infant formula by liquid chromatography-quadrupole linear ion trap mass spectrometry. Journal of Dairy Science; 9: 679-686.

6. Timotheou-Potamia M, Calokerinos AC. 2007 Chemiluminometric determination of vanillin in commercial vanillin products. Talanta; 71: 208-212.

7. Palanisamy S, Kokulnathan T, Chen S-M, Velusamy V, Ramaraj SK. 2017. Voltammetric determination of Sudan I in food samples based on platinum nanoparticles decorated on graphene$\beta$-cyclodextrin modified electrode. Journal of Electroanalytical Chemistry; 794: 64-70.

8. Kumar AA, Swamy BEK, Rani TS, Ganesh PS, Raj YP. 2019 Voltammetric determination of catechol and hydroquinone at poly (murexide) modified glassy carbon electrode. Materials Science \& Engineering $C$; 98: 746-752.

9. Shahrokhian S, Hafezi-Kahnamouei M. 2018. Glassy carbon electrode modified with a nanocomposite of multi-walled carbon nanotube decorated with $\mathrm{Ag}$ nanoparticles for electrochemical investigation of Isoxsuprine. Journal of Electroanalytical Chemistry; 825: 30-39.

10. Manasa G, Mascarenhas RJ, Satpati AK, D'Souza OJ, Dhason A 2017. Facile preparation of poly(methylene blue) modified carbon paste electrode for the detection and quantification of catechin. Materials Science and Engineering C; 73: 552-561.

11. Brett CMA, Inzelt GÈ, Kertesz V. 1999. Poly(methylene blue) modified electrode sensor for haemoglobin. Analytica Chimica Acta; 385: 119-123.

12. Sun W, Wang Y, Zhang Y, Ju X, Li G, Sun Z. 2012 Poly(methylene blue) functionalized graphene modified carbon ionic liquid electrode for the electrochemical detection of dopamine. Analytica Chimica Acta; 751: 59-65.
13. Chen H, Zhang Z, Cai D, Zhang S, Zhang B, Tang J, Wu Z. 2012. Attapulgite with poly(methylene blue) composite film:Electrocatalytic determination of ascorbic acid. Solid State Sciences; 14: 362-366.

14. Dilgin Y, Canarslan S, Ayyildiz O, Ertek B, Nişli G. 2012. Flow injection analysis of sulphide based on its photoelectrocatalytic oxidation at poly-methylene blue modified glassy carbon electrode. Electrochimica Acta; 66: 173-179.

15. Ahmed AM, Sayed SY, El-Nagar GA, Morsi WM, El-Deab MS, El-Anadouli BE. 2019. Enhanced electrocatalytic oxidation of glucose at graphene nanosheets-Metal oxides nanoparticles modified GC electrodes. Journal of Electroanalytical Chemistry; 835: 313-323.

16. Arslan E, Cakır S. 2016. Electrochemical fabrication of polyproline modified graphite electrode decorated with $\mathrm{Pd}-\mathrm{Au}$ bimetallic nanoparticles: Application for determination of carminic acid. Journal of Electroanalytical Chemistry; 760: 3241.

17. Daneshvar L, Rounaghi GH, Es'haghi Z, Chamsaz M, Tarahomi S. 2016. Fabrication a new modified electrochemical sensor based on $\mathrm{Au}-\mathrm{Pd}$ bimetallic nanoparticle decorated graphene for citalopram determination. Materials Science and Engineering $C$; 69: 653-660.

18. Shang L, Zhao F, Zeng B. 2014.Sensitive voltammetric determination of vanillin with an AuPd nanoparticles-graphene composite modified electrode. Food Chemistry; 151: 53-57.

19. Koçak ÇC, Karabiberoğlu Ş. 2018. Electrochemical Vanillin Determination on Gold Nanoparticles Modified Multiwalled Carbon Nanotube Electrode. Dokuz Eylul University-Faculty of Engineering, Journal of Science and Engineering; 20(59): 461470.

20. Huang L, Hou K, Jia X, Pan H, Du M. 2014. Preparation of novel silver nanoplates/graphene composite and their application in vanillin electrochemical detection. Material Science and Engineering $C$; 38: 39-45. 